\title{
El cuento de humor: su identidad cultural en San Pablo de Yao, Cuba
}

\author{
The humorous story: their cultural identity of San Pablo de Yao, in \\ Cuba
}

Lohema Céspedes Ginarte Icespedesg@udg.co.cu

Universidad de Granma (Cuba)

\section{Resumen}

Al ser la comunidad un espacio intermedio entre la familia y la sociedad comporta ventajas importantes para la realización de un estudio de las ciencias sociales. El cuento de humor: nuestra vida en las bocas. Un estudio sociocultural sobre la identidad cultural de San Pablo de Yao, en Buey Arriba, Granma es uno de esos estudios que permean las comunidades cubanas. A partir de tratamientos teóricos conceptuales de las categorías identidad cultural, oralidad, tradiciones orales y cuento de humor se obtuvo que este estudio demostró la validez de la perspectiva sociocultural para el análisis del cuento de humor como manifestación oral concreta en comunidades rurales y el alcance de este en su identidad cultural; la descripción comparativa de los cuentos de humor permitió el estudio de sus variaciones en relación con parámetros demográficos como sexo, edad y nivel de instrucción; la comprensión de la trascendencia en términos de identificación cultural; y se recogió la valoración de las informaciones obtenidas. Religión, raza, muerte, los relacionados con la fauna, matrimoniales, 
orientación sexual y problemas de salud constituyen temáticas recurrentes en San Pablo de Yao donde se encuentran las claves para entender el ser de sus portadores y cultores.

Palabras clave: Identidad cultural; oralidad; tradiciones orales y cuento de humor.

Abstract

As the community is an intermediate space between the family and society, it has important advantages for carrying out a study of the social sciences. The humorous story: our Life in the mouths. A sociocultural study on the cultural identity of San Pablo de Yao, in Buey Arriba, Granma, is one of those studies that permeate the Cuban communities. Based on theoretical conceptual treatments of the categories cultural identity, orality, oral traditions and humor, it was found that this study demonstrated the validity of the sociocultural perspective for the analysis of the humor story as a concrete oral expression in rural communities and the scope of this in its cultural identity; The comparative description of humor stories allowed the study of their variations in relation to demographic parameters such as sex, age and level of education; the understanding of transcendence in terms of cultural identification; and the evaluation of the obtained information was collected. Religion, race, death, those related to fauna, marriages, sexual orientation and health problems are recurring themes in San Pablo de Yao where the keys to understanding the being of its bearers and cultists are found.

Keywords: Cultural identity; orality; oral traditions and humor.

Muchos han sido los estudios dedicados a la necesidad de la identidad, con aproximaciones de autores contemporáneos de enfoques, maneras y perspectivas muy diversas. Basta mirar alrededor para darse cuenta que, hoy en día, constantemente estudiosos de la talla de Nara Araújo, Fernando Ortiz (1993), Carolina de la Torre, Gregorio Hernández Zamora, Maritza García y Cristina Baeza (1996), entre otras grandes personalidades de los estudios identitarios no solo en el ámbito nacional, sino también a escala internacional, entran en contradicción por dicha temática. No se trata únicamente de una necesidad cognitiva y práctica, es también una necesidad existencial.

Algunos de estos investigadores discuten sobre el intento casi estéril de reducir en pocos renglones un proceso tan abarcador y significativo como el de la identidad en su sentido mayor 
de generalidad, prefiriendo en su lugar aplicar el concepto a campos más específicos, según sea el objeto de estudio y la rama del conocimiento en que se inscribe la investigación; decisión con la que se coincide.

La amplitud cosmovisiva del término sugiere un enfoque multilateral de carácter permanente y, para su estudio, no se pueden perder de vista sus manifestaciones concretas en el devenir social. Tales razones, necesariamente, implican una dimensión cultural en su tratamiento epistemológico, pues es la cultura su medio de expresión y constitución más directa.

En nuestra opinión, la identidad es un resultado necesario del desarrollo de las relaciones sociales, es expresión de estas y se construye en la propia participación social, se articula como componente básico de la realidad no solo objetiva sino subjetiva, donde en una red de vínculos con los demás los individuos internalizan los valores y normas básicas de la sociedad que viven.

Esto se materializa como objetivación práctica en su vínculo con la cotidianidad y, más directamente, en las producciones culturales de los sujetos sociales en actividad colectiva, cuya plataforma medular radica en la subjetividad humana. De ahí que entender la identidad cultural como fenómeno social consigna un análisis desde una óptica transdisciplinaria que unifique los retos de la Psicología, la Antropología, la Sociología, la Filosofía y otras muchas ciencias que han incluido en sus perfiles de trabajo la búsqueda de una definición categórica del término.

Orozco (1994), en una síntesis entre las grandes direcciones del pensamiento cultural, contribuye a la construcción de una imagen más integradora y completa de la realidad humana contemporánea cuando asume que "es conciencia de unidad en sí mismo y de pertenencia a determinados espacios sociales que alcanza un sujeto cultural como resultado de un proceso interactivo y de comunicación social, psicológica, e histórica con otro(s) sujeto(s) cultural(es)" (p. 4).

La anterior conceptualización registra como propiedad derivada del fenómeno identitario al sentimiento de pertenencia, concibiéndose como "la capacidad desarrollada por los comunitarios para asumir, promover y defender los valores propios de su comunidad. Sentimiento de orgullo con el que se expresa la historia viva de su barriada, como la razón de ser" (Castellanos, 2010: 24).

Visualizada desde estas orientaciones, la identidad es un constructo dinámico y jerarquizado que se desarrolla en un proceso de construcción y deconstrucción, en términos de individuos, grupos y comunidades humanas. Su ubicación en un contexto geográfico, político, cultural, 
económico, histórico, muestra lo ilógico de un producto estático. La identificación siempre lía cambios, ensanchamientos y adaptaciones según diferentes tejidos sociales, en un devenir de continuidad y discontinuidad, de unidad y diferencia.

Es precisamente en esa múltiple integración de referentes diversos, lleno de vicisitudes y complejidades propias del entorno histórico-social y físico-natural, que se van produciendo las mezclas, préstamos y elaboraciones sintéticas que definen lo que, para cada momento contextual, presenta la cualidad de determinada identificación cultural.

Ortiz (1993: 90) expone que es "resultado de la conjugación de necesidades, aspiraciones, medios, ideas, trabajos y peripecias de sus componentes diversos". Por su parte, Monal (2001: 551) "parte del supuesto de comprender la identidad como una totalidad orgánica compleja, que expresa la realidad sociocultural en sus estructuras, nexos y complejidades", lo que se constituye cardinal a los efectos de este estudio.

Es importante considerar aquí al individuo y al grupo humano concretos, que funcionan en un medio sociocultural específico de normas, valores y un sistema de instituciones y esferas de actividad, en los que asumen responsabilidades, compromisos y roles sociales.

Reflexionar acerca de la necesidad humana de saber cómo somos, quiénes somos, de dónde venimos, hacia dónde vamos y de qué grupos sociales nos sentimos parte, no aleja la evidencia objetiva, pero se adelanta la dimensión subjetiva, o sea, la importancia del sentimiento de pertenencia como componente de la identidad cultural y como elemento regulador del comportamiento, en la medida en que motivaciones, sentimientos, prejuicios y actitudes, más o menos continuos, se deben expresar en actuaciones congruentes con ellos.

En una contemplación mucho más integradora se encuentran otros apuntes de investigadoras sociales, que recogen en apretada síntesis, pero en extensión de contenido, los principales componentes del fenómeno tratado:

\footnotetext{
llámese identidad cultural de un grupo social determinado (o de un sujeto determinado de la cultura) a la producción de respuestas y valores que como heredero y trasmisor, actor y autor de su cultura, el grupo social determinado o el sujeto determinado de la cultura realiza en un contexto histórico dado como consecuencia del principio sociopsicológico de diferenciación-identificación en relación con otro (s) grupo (s) o sujeto (s) culturalmente definido (s) (García y Baeza, 1996: 12).
}

Este enfoque aborda un tratamiento dialéctico al fenómeno identitario, pues asume al individuo como componente de grupos y de una sociedad que posee una estructura social y cultural 
determinadas, a la cual se adapta mediante la socialización de normas y responde en consecuencia con la significación que adquiere en la persona, facilitando la adquisición de una imagen propia y una singularidad identificadora.

Así, la identidad no está ahí para ser manifiesta solamente, sino para ser reconocida y aceptada en un proceso práctico y comunicativo, donde participan siempre dos extremos: los sujetos de la identidad y los otros que la reconocen, aceptan o rechazan. La misma tiene lugar en la actividad y la comunicación humana por potentes mecanismos de interacciones sociales, donde unos y otros asumen un papel dinámico.

Como resultado de la tipificación e interpretación de los componentes y atributos puestos a consideración, se asume la identidad cultural como:

\begin{abstract}
proceso histórico-cultural concreto de carácter sociopsicológico, cuya constitución tiene lugar en un contexto específico de relaciones sociales, donde se refuerza la diferenciaciónfiliación de los grupos sociales a partir de su concepción del mundo, se fija la atención en los rasgos culturales que los caracterizan y diferencian de los otros, y se subraya una modalidad de lengua determinada, que posibilita valorar el lenguaje como parte importante de la realidad sociocultural de los hombres y las colectividades a las que pertenecen (Castellanos, 2010: 18).
\end{abstract}

Queda claro aquí que la identidad cultural remite al sentimiento de pertenencia y señala un inherente carácter histórico, constatación que establece un punto de partida teóricometodológico y orienta hacia un modus operandi en la dinámica de adentrarse en su estudio.

Dicha postura está dotada de esclarecimiento gnoseológico, pues ubica la comprensión estructural y las relaciones de sus partes (de la identidad cultural) intransitable al conocimiento fuera de los márgenes interdisciplinarios. Establece la necesidad de una cooperación armónica fecunda que implica mucho más que una simple yuxtaposición de diversas disciplinas, es decir, la producción de categorías que correspondan a un conjunto orgánico, tomando como iniciativa la inclusión de la palabra hablada como componente vertebral en dicho proceso.

Asimismo, se valora el lenguaje como un fenómeno sociocultural que posibilita estudiar los grupos humanos y sus relaciones sociales, específicamente en el discurso oral; lo que impone entenderlo como un instrumento comunicativo ajustado al entorno social que rodea su realización, valorándose así el lenguaje mismo como un acto social; a la vez que modula los valores culturales de los sujetos que los identifican, se reflejan y ejecutan a través de la lengua. 
La dinámica de esta construcción teórica incluye el proceso de reconstitución, o sea, una restauración y composición que, sin embargo, le permite a la identidad mantener y conservar las constantes de sus ingredientes y elementos originarios con aquellos incorporados, adquiriendo arraigo y permanencia en ella por tratarse de actividades y manifestaciones concurrentes, participativas.

Se trata de lazos históricos expresados a través de prácticas funcionales. De ahí vienen y se acumulan relaciones, y al prolongarse se vuelven costumbres, comportamientos que de extenderse tanto se hacen comunes. Combinados, interrelacionados, fusionados en recreaciones beneficiadas en sus esencias primigenias, se convierten en tradición; tradición que pasa de generación en generación a través del mecanismo de la comunicación, donde la oralidad desempeña el papel cardinal.

\section{Categoría sociocultural de voluntad testimonial: la oralidad}

Multiplicidad de enfoques se aplican al estudio de la oralidad: desde el histórico hasta el idiomático, pasando por el etnográfico, el antropológico, el sociolingüístico, las ciencias de la información y otros de más reciente desarrollo y con una novedosa propuesta de aproximación a una importante cuestión relativa a los estudios socioculturales, los que reconocen el valor que posee la memoria cultural conservada mediante la repetición oral de temas claves y las peculiaridades de una sociedad específica con su traslado continuo y multiforme de generación en generación.

En las décadas más recientes, dicho fenómeno ha entrado en usanza cada vez más familiar, aun en esferas que inicialmente no habían mostrado ningún interés al respecto. En este sentido, entran en popularidad términos como literatura oral, tradición oral, narración, lenguaje, discurso hablado, etc., no solo para los estudiosos de la cultura, del lenguaje y la comunicación, sino igualmente para la intelectualidad genérica más o menos consciente de los temas que maneja.

El sustento y la fijación de formas de comportamiento comunitario e individual, su historia, sus leyes, sueños y creencias, formas de alimentación e higiene, métodos de curación, fórmulas laborales, estilos de relaciones interpersonales y sexuales, entre otros, han recaído sobre los hombros de la oralidad. 
Fue entonces, durante largo tiempo, el único sistema de expresión de hombres y mujeres y también de transmisión de conocimientos y tradiciones integrantes de la identidad cultural de las regiones, con mucha más fuerza en las zonas rurales. Ella constituye así, un sistema simbólico de expresión, un acto de significado dirigido de un ser humano a otro u otros, y es quizás la característica más significativa de la especie.

Desde presupuestos etnológicos, lingüísticos, sociológicos, trascienden prolíferas interpretaciones de dicho fenómeno social, que si bien son acertadas - 0 al menos ajustadaspara su contorno epistémico, aún resultan limitadas para análisis multidisciplinarios de los cuales demanda la realidad actual.

La comunicación oral precisa un estudio con carácter integrador, que profundice mucho más en relaciones que se dan a su interior y no solamente en descubrir mecanismos externos que influyen o componen dicho proceso, razón por la cual, con ajustada certeza se plantea:

\footnotetext{
El mundo de lo oral merece ser apreciado desde su estrecha relación con las actitudes, acciones, relaciones más consustanciales del ser humano, como lo propio de él. La oralidad encierra desde la palabra como reguladora ética de los grupos humanos, hasta los matices de las expresiones, sus relaciones con los sentimientos, acciones, costumbres de los individuos, donde no se pueden perder de vista los fenómenos de transmisión- apropiación, recepción-voz-silencio, gestos, ritmo, aspectos que no han sido estudiados por muchas disciplinas (Rodríguez Cosme, 2008: 13).
}

Lo que acontece en determinados contextos y situaciones en relación con el habla, puede superar la instantaneidad y lo efímero, pues su conocimiento se recibe y se conserva gracias a la transmisión de la memoria histórica que trasciende de una época a otra, demostrando que la oralidad es dinámica, porque se mueve, trasforma, se enriquece a través del tiempo y logra establecer lazos comunicantes entre los individuos.

De aquí se desprende una lógica epistemológica que indica, en un primer instante, que la oralidad constituye un sistema de códigos y mensajes analíticamente inseparables de su entorno y dotados de una dependencia relativa y; en un segundo momento, que dicha oralidad se inserta en la casi totalidad de los hechos humanos, con los cuales interactúa constantemente dando origen a una influencia mutua y creativa, aunque sí presenta características propias y específicas que le confieren una dimensión muy peculiar y sui géneris en el contexto de cualquier cultura.

Al respecto, resulta significativo señalar otra postura científica cuando se asume 
que la expresión oral es siempre un proceso que se produce en el intercambio de realizaciones concretas determinadas, a través de la transmisión, recepción de pensamientos ofrecidos por la voz convertida en palabra. También constituye la transmisión de un legado histórico o de una memoria (Victori Ramos, 2004: 15).

De esta forma, el espacio de lo oral es producción cultural permanente y depósito de la memoria colectiva, mientras que la comunidad (ente socio comunicativo) funciona como productora y árbitro de los cambios que se dan dentro de la práctica cotidiana, selecciona, acepta y fija en la memoria, porta y transmite aquellos contenidos que le son necesarios para el desarrollo de su propio entorno.

Como acto de la lengua, arte verbal, repertorio de códigos y sistemas expresivos de la comunicación, la oralidad constituye entonces una fuerza socio histórica colosal de dimensión sígnica; además, tiene una propiedad inherente que permite la manifestación de consideraciones implícitas como parte de los contextos situacionales, sociales y culturales que dan sentido a lo expresado.

Como proceso de comunicación, se encuentra vinculado a la construcción de significados mediante el cual los sujetos articulan interpretaciones acerca de fenómenos constitutivos de la sociedad -el poder, la historia, la cultura, la ideología, la política- y ponen de manifiesto diferentes formas de representación social de la realidad.

La transmisión constituye el mecanismo del cual se ha valido lo expresado para alcanzar perdurabilidad histórica y criterios válidos aceptados por sus aportes teóricos al estudio de dicho fenómeno. Los mismos apuntan a que la transmisión

\begin{abstract}
permite la continuidad en el conocimiento de los valores primarios en que se asienta la identidad cultural de una comunidad dada, pasa la concepción de su ethos social de una generación a otra", y añade como un segundo mecanismo de relevancia connotativa la recreación, "porque cada sociedad recrea sus valores en un proceso de adecuación a la realidad histórica que se vive (Cordiés Jackson, 2001: 1).
\end{abstract}

Esta relación transmisión-recreación posibilita registrar las vivencias más sensibles y sustanciales del ser humano y de las comunidades y grupos que él integra, pues en la memoria solo se fijan los actos relevantes a partir de la significación que alcancen en grado individual y colectivo; cuestión que apunta necesariamente a la existencia de un tercer proceso de especial 
importancia: la recepción, pues todo sistema de comunicación implica -en función de lograr el intercambio- la comprensión, asimilación y admisión de la palabra estructurada en mensaje. Las valoraciones realizadas hasta este momento de la investigación infieren una interpretación obligada del fenómeno oral en interrelación con el contexto sociocultural, de donde emerge y el cual deviene receptáculo de dicha expresión oral, pues en ellas se explicitan tanto las cosmovisiones de los sujetos sociales y sus grupos, como un sinnúmero de actitudes, motivaciones, expectativas, saberes, etc., que trascienden el marco socio histórico.

Ello tiene como mediación el proceso del intercambio comunicativo, que implica

no solo el cuidado en el uso de la lengua, sino un respeto al turno de la palabra, a la escucha activa, al cumplimiento de la entonación, las pausas, el ritmo, la intensidad, el silencio, que junto con los elementos no verbales, posibilitan asimilar los saberes lingüísticos del habla (Rodríguez Cosme, 2008: 46).

Ello se considera un aporte de marcada relevancia para las ciencias sociales a pesar de su interpretación pedagógica al fenómeno oral. Entendiéndose aquella como el conjunto de mitos, leyendas, cuentos, adivinanzas y décimas que resumen saberes, modos de vida reflejados a través de un contenido significativo y didáctico del que se apropian los sujetos en la comunicación oral.

Los autores del presente estudio, asumen dicha definición amén de resultar limitante la referencia de forma cerrada de las manifestaciones inclusivas de la oralidad, dejando fuera algunas expresiones del fenómeno oral como pregones, fábulas, refranes, ensalmos, agüeros, conjuros, etcétera.

\section{La tradición oral: nuestra vida en las bocas}

La tradición oral se constituyó como el vehículo más utilizado para trasladar conocimientos y experiencias y hoy en día aparece acompañado de la memoria histórica, creación colectiva cuya función consiste en preservar y ordenar los conocimientos mínimos indispensables para asegurar la supervivencia del grupo. En síntesis, se trata de acumular experiencia humana y transmitirla con precisión a las generaciones futuras.

Dentro de este análisis demanda a su favor resaltar la inclusión de aspectos como la cultura popular y la identidad de los sujetos portadores de dicha tradición, pero resulta limitante la poca 
importancia que se le confiere al contexto en el cual se generan las mencionadas prácticas comunicativas.

Por eso, se precisa llevar a cabo un estudio interpretativo de esta problemática, donde no solo se preste atención a lo expresado, sino a las condicionantes socioculturales que dan sentido a lo enunciado, pues una misma manifestación oral en ambientes diferentes de desarrollo contiene cargas simbólicas y significaciones distintas, alcanzando o no la trascendencia en el tiempo.

La tradición, en este sentido, constituye una forma de redefinición continua de todos aquellos valores, creencias y prácticas cotidianas que preservan las culturas y las comunidades de la degradación del olvido.

De esta manera, una forma de narrar lo constituye el cuento popular o tradicional, este último de vital importancia para las Ciencias Sociales por constituir una expresión identitaria pura y por su carga simbólica cultural. Sin embargo, las realidades socioculturales son nuevas y con nuevas interpretaciones hasta para contarlas, por lo que se ha gestado un cambio sustancial en la forma genérica de narrar las historias colectivas o personales.

El cuento oral en su expresión popular colectiva encierra en su corpus un sin número de creencias, ritos, costumbres, etcétera; por tanto, depende del medio social en que se produce, resultando históricamente transitorio.

En el caso particular de Cuba, fundamentalmente en las zonas rurales como San Pablo de Yao, los cuentos populares de humor, gozan de una preferencia de exposición, ya sean de temas humanos o animales, matrimoniales, religiosos, raciales, geográficos, etc., pues no requieren de una preparación o una ambientación especial. Las exposiciones son entonces incidentales, informales y espontáneas. Estos temas son recreados de forma rutinaria con acontecimientos y vivencias de la vida en colectividad, son expresión de todo un cúmulo de saberes forjados en la propia interacción social, donde la percepción que se adquiere del mundo luego se ve manifiesta en dichas creaciones socioculturales.

Ello alude a la forma y al contenido de los relatos con orientación cómica, pero que pone por encima de toda estructura posible del cuento, las habilidades del narrador, pues no es suficiente con un buen esquema y una magnífica historia para relatar; se necesita mucho más que eso, se necesita a decir de buen cubano haber "nacío pa'eso".

El cuento oral como construcción social no solo existe en la conciencia del sujeto, sino en todo el espacio de su cultura. $Y$ como complejo del saber pragmático se fija en la conciencia, la voluntad y los sentimientos, el lenguaje, los objetos y la propia actividad humana; con su fuerza 
ideal activa, como modulador de las formas de percepción y representación, de sentimientos y voluntad, como imperativo hacia la acción. Por tanto, en su vertiente humorística, resulta privilegiado en el oficio de comunicar historias reales o imaginadas, posee condicionamientos y expectativas propias, que nunca la escritura (transcripción), por más que se acerque, podrá homologar. Solo al mundo de la oralidad le está conferido tal privilegio.

Resulta imposible hablar de cuentos de humor y tradición oral sin hacer referencia obligada al campo de la cultura, de donde se gestan y derivan todas estas manifestaciones que constituyen, por antonomasia, la cultura popular tradicional de una zona específica, pues tanto su aspecto popular como tradicional resultan del proceso de comunicación y vida societal, los cuales derivan con el transcurso del tiempo, en expresión tipificada de la cultura de los sujetos de dichas comunidades humanas, o lo que resulta equivalente, la identidad cultural de los citados individuos.

Resumiendo estos conceptos y todas las peculiaridades antes mencionadas, el cuento es un breve relato, ocupado por un hecho central pero no exclusivo, en forma sencilla, graciosa, original e interesante de un acontecimiento ficticio o real, con predominio de fantasía e imaginación, capaz de excitar, impresionar y provocar la risa como síntoma de buena recepción en el público, con una carga identitaria por excelencia sintetizando la cosmovisión y las características socioculturales del portador de la tradición.

\section{Valoración del cuento de humor por los habitantes de San Pablo de Yao}

A partir de los resultados obtenidos en la comunidad montañosa de San Pablo de Yao, perteneciente al municipio Buey Arriba, provincia Granma, se pudo apreciar la significación que reconocen los habitantes acerca del cuento de humor como tradición oral de marcada presencia en su ámbito cotidiano. Fueron objeto de estudio diez personas, quienes se entrevistaron en su propio escenario y no presentaron dificultades con la expresión. Se logró así una comunicación natural y espontánea y se enunciaron algunas ideas de gran importancia, las cuales aparecen recogidas a continuación, teniendo en cuenta los grupos etáreos:

Grupo etáreo I (de 20-35 años) 
Los cuentos analizados son una muestra de la jocosidad del lugareño de San Pablo de Yao. Con sistematicidad aparecen palabras y frases puntuales con diferencias en su pronunciación y significación, las cuales permiten visualizar el humor característico de la localidad.

En este primer grupo, jóvenes todos, se evidencia la asimilación de esa tradición colectiva, así como la insistente pronunciación exacta de las diferentes palabras con omisiones (terminao, colorao, preparao); apócopes (na, usté, ve, má, prepará, año); empleo de términos foráneos (jistory, dog, chopins), eufemismos (lo mismo, pero con tostones) y cambio de letras (güevos, la cousa), así como la reminiscencia a los cuentos infantiles clásicos (había una ve) que dan lugar a un modo distinto de contar, pero sobre todo, sin perder la idea de lo contado.

En relación con el aspecto contextual aparecen evocaciones al entorno local como las frases: dicen que fue aquí donde pasó, una ve aquí vinieron, hicieron la reunión en la mata de almácigo, los juicios del pueblo, ahí en la escuela de La Nave, entre otras que muestran el arraigado tradicional y la contextualización que existe en los cuentos de humor.

Resalta también en este grupo la estructuración narrativa donde, de forma ingenua, se presentan los personajes representativos (Pepito y Marcelo) de aventuras, atrevimientos, fechorías, travesuras y cuanta acción descabellada nazca del ingenio colectivo, las acciones y el desenlace de igual manera en la mayoría de los casos. Ello denota una fijación normativa del habla y la extensión de las estrategias organizativas internas en los cuentos de la zona.

Desde el punto de vista temático, es meritorio destacar la presencia de argumentos vinculados con las relaciones amorosas y sexuales, los aspectos legales, profesionales, docentes, alimenticios y situaciones monetarias, entre otras, que dan fe de las preocupaciones e inquietudes del grupo etáreo al que pertenecen.

De manera encubierta asoman valoraciones, juicios críticos y argumentaciones que señalan el complejo sistema de valores e ideología que rige el comportamiento de los pobladores locales, así como la significación social alcanzada por algunos fenómenos censurados mediante la comicidad. Esto explica el contenido educativo de estas expresiones orales, las que fomentan a su vez, prácticas sociales con carga formativa y patrones conductuales acordes a la ética de los moradores. Por ende, descuella el cuento humorístico como un instrumento de lecciones púdicas.

Grupo etáreo II (de 36-60 años)

En este segundo grupo sucede algo similar al del anterior, pues se centra en cuestiones donde el apócope (pa, tra, cantida, pa, ve); la omisión (cansao, llevao, desgraciao); la reminiscencia a 
los cuentos infantiles (hay una ve) se mantienen. Igualmente, lo risible y lo jocoso complementan la ingeniosidad del cuentero y, de cierta manera, logran la credibilidad de los mismos. Se evidencia además, en este segundo grupo, un recurso estilístico nuevo y se trata de la adición de letra: manicornio por manicomio.

Desde el ángulo estructural resulta oportuno aludir a la forma genérica de disponer los elementos que conforman la narración, tanto los protagonistas, las acciones como los finales, coinciden con las anteriores descripciones. Se muestra entonces la generalización distributiva y aspectos afines en este sentido.

Los tópicos que abundan en esta generación tocan aspectos puntuales de la vida como las suegras, los negros, los locos, las relaciones de pareja, temas religiosos, de animales e, inclusive, afloran figurantes alcohólicos. Ello denota cómo la realidad que se vive en la localidad permea estas narraciones orales y la utilización de la jocosidad para encubrir el tratamiento de los problemas que inquietan a la comunidad.

A la par, se evidencia una certera crítica de los males sociales que se enfrentan en la actualidad, desde una postura que no los compromete y que, sin lugar a dudas, les permite disfrutar y entretenerse. A la vez, emiten valoraciones y logran tasar la realidad sociocultural de los propios pobladores rurales. Se manifiesta además una finalidad instructiva, pues tales expresiones orales encierran en su interior un sistema de valores y una norma social de actuación, armónica siempre con las exigencias de la sociedad.

Es menester señalar que, durante el examen del discurso oral de estos hablantes, se constató la alusión directa al área particular, a través de locuciones como: la iglesia de aquí, la gente del pueblo, había aquí un hombre y allá afuera. Todas ellas con la intención bien marcada de hacer la historia que se cuenta más original y a la vez más suya. Así se manifiesta el sentido de pertenencia hacia su comunidad y jactancia de vivir en una localidad como San Pablo de Yao.

Grupo etáreo III (de 61 en adelante)

Aquí se evidencia un grupo de palabras y frases que permiten la presencia de la identidad a través del habla. Las palabras subrayadas distinguen el proceso del hablante para referirse a algo en específico, propio de una zona rural. Se muestra además el uso incorrecto de las mismas, sin conducir en lo absoluto a ambigüedades ni que ello constituya algo ininteligible.

Próstrata, herradía, güevos, reenlámpago, el bati blanco, zunzún, tarto rectal, le mira el cuero, análises, tan tan tan, varios nortes del cuerpo, son palabras que infieren, de hecho, los temas por los cuales transita el narrador de cuentos residente en San Pablo de Yao: religiosos, 
raciales, de muerte, relacionados con la naturaleza, matrimoniales, de orientación sexual y hasta problemas de salud se manifiestan de forma heterogénea.

Estos son, al igual que los ejemplos anteriores, casos donde el tema en cuestión, la manera de emplear los términos, la omisión de sonidos, la jocosidad del cubano y las palabras puntuales de una persona natural del campo, dan fe del tratamiento dado a una historia que fue desde sus inicios bien relatada. Los gestos de las manos, del rostro cobran una significación sustancial para ser mucho más creíble esa historia imaginada por un oriundo y residente de San Pablo de Yao.

También, en estas narraciones orales se dilucidó la sugerencia al ámbito cotidiano mediante expresiones como: de la Nave de Yao, aquí una vez, al puestecito de vender, la estación de herradía de animales y boeyes de quí mismito, era de aquí, aquí mismo en Yao, es gente de aquí mismo, entre otras. Por ende, se exponen patrones de contextualización que ilustran lo paisano de estas historias y conmoción por parte de los serranos de Yao hacia su lugar de convivencia.

Lo estructural, por su parte, se concreta en los hablantes de la comunidad objeto de estudio a través de la mayoría de sus discursos, donde se colocan de forma homóloga a los anteriores cuentos explicitados, todos los componentes de dichos sucesos. Ello explica la lógica evolutiva que rige la organización interna de estos cuentos de humor, en los cuales de manera ordenada se sitúan los personajes, las acciones que estos protagonizan y el final de las situaciones que se enfrentan.

Según el análisis dispuesto, se presencian evaluaciones y sátiras, así como la repercusión e importancia de algunas temáticas que asumen los pobladores de San Pablo de Yao como materias de interés para ser abordadas desde la óptica burlesca. Ello implica una dimensión educativa, pues marca líneas de comportamiento social entre los habitantes de este paraje, quienes por temor a ocupar una posición ridícula mantienen un proceder conforme a lo reglamentado por sus propias leyes de actuación social. Así, el cuento de humor se convierte en un medio eficaz para la enseñanza, la toma de lecciones y un vehículo de didáctica social.

\section{A modo de conclusión}


Los presupuestos teóricos que sustentan la investigación relativa a la identidad cultural y la oralidad posibilitaron su definición como fenómenos socioculturales. En tanto, en San Pablo de Yao, como contexto sociocultural compartido por los sujetos investigados, se signó la descripción comparativa de los cuentos de humor y ello permitió el estudio de sus variaciones en relación con parámetros demográficos como sexo, edad y nivel de instrucción; así como la comprensión de la trascendencia en términos de identificación cultural.

Se pudo obtener información acerca del comportamiento sociocultural de los pobladores y se logró compilar conocimientos elementales e indispensables para el desarrollo de técnicas posteriores. La misma fue aplicada indistintamente a los tres grupos etáreos que se establecieron, lo que permitió determinar los informantes claves para dicho estudio, así como describir el comportamiento empírico-pragmático de la tradición oral en cuestión y el sistema de relaciones sociales que a su alrededor se desenvuelven.

Adyacente al empleo de esta práctica investigativa se utilizó como procedimiento de trabajo, para el registro de la información derivada del contacto social con los moradores de la localidad, las grabaciones encubiertas y notas de campo, ambas complementando los datos recopilados durante la observación participativa. Ello permitió a través de las grabaciones, obtener los cuentos de humor en su contexto natural de proliferación y la toma de apuntes básicos, que resultaron medulares e implicó una medida de precaución en lucha contra el fenómeno del olvido y con lo efímero de los acontecimientos sociales.

En consecuencia con esta fusión técnica, se obtuvo como resultado parcial una selección adecuada de informantes ideales y representativos en relación con el culto del cuento de humor; un diagnóstico detallado del acontecer de la comunidad desde una perspectiva sociocultural y la descripción del cuadro de relaciones sociales que trascienden en dicha colectividad.

El análisis de contenido propuesto constituye uno de los aportes principales del estudio que se presenta, y posibilitó la determinación de las características básicas de los cuentos de humor de los pobladores de San Pablo de Yao.

\section{Bibliografía}

Baeza, C. y García, M. (1996). Modelo teórico para el estudio de la Identidad. La Habana, Cuba: Editorial José Martí. 
Castellanos, Y. R. (2010). La perspectiva lingüístico-antropológica en tradiciones orales como expresiones de identidad. (Tesis doctoral). Universidad de Oriente, Santiago de Cuba.

Cordiés Jackson, M. (2000). Oralidad y Liturgia. Centro Africano "Fernando Ortiz". Editorial Santiago, Santiago de Cuba.

Monal, I. (2001). Identidad: Entre Inercia y Dinámica. El acecho de la razón identitaria pura. En Filosofía y Sociedad. La Habana: Editorial Félix Varela.

Ortiz, F. (1993). Etnia y sociedad. Temas, 28, Editorial Ciencias Sociales, La Habana, pp. 8792.

Orozco, I. (1994). Apuntes para una definición de la identidad. Granma, Cuba: Ediciones Bayamo.

Rodríguez Cosme, M. (2008). La oralidad, una vía para el desarrollo de la competencia comunicativa en los escolares rurales del segundo ciclo de la escuela primaria rural. (Tesis doctoral). Instituto Superior Pedagógico Frank País García, Santiago de Cuba.

Victori Ramos, M. del C. (1999). Lo oral en la encrucijada. En Vera, A. La Oralidad ¿ciencia o sabiduría popular? (pp.32-41).La Habana, Cuba: Editorial Linotopia Bolívar y Liturgia. 


\section{Anexo 1}

\section{Cuentos de humor del grupo etáreo I}

\section{(25 años)}

Aquí había un macho que se empató una ve con una señora toque/ no tan pura pero ya con años en la costilla/ pero se mantenía muy bien ella// La señora muy culta/ fina y prepará/ y con piticliny/ lo invitó a una cantina de un restauran de altura/ y llega con el tipo/ que lo vistió y lo calzó ya tú sabe/ con pantalón de hilo/ camisa de manga por dentro/ ¡fino!, te cuento que había que verlo pa' creerlo/ llegaron al bar y viene el socio y le dice:

-Buenas noches/ ¿qué desean consumir?//

Dice ella:

-Un mojito cubano/ con limón//

- ¿y usted?//

-Yo/ lo mismo/ pero con tostones//

\section{Anexo 2}

\section{Cuentos de humor del grupo etáreo II}

(38 años)

Está una ve el cura de la iglesia de aquí predicando/ está dando la misa con la gente del pueblo y tiene una pelotica y está diciendo: hijos míos/voy a tirar esta pelotica y al que le dé en el pecho/ es el más pecador del pueblo// Y el cura coge y tira la pelotica y en la iglesia había un ventilador y vira pa' tra y le da al cura en el pecho y ahí el cura dice: de rebote no vale//

\section{(41 años)}

Llega la mujer de Marcelo de la calle y está el marido en el sofá borracho como casi siempre y la mujer le dice: tengo una noticia muy mala que darte papito/ a partir del día 1ro. el ron va a valer tres veces más de lo que vale ahora/ el borracho la mira y le responde/ yo también tengo una noticia muy mala que darte/ a partir del día 1ro. vas a pasar un hambre del carajo//

\section{Anexo 3}

\section{Cuentos de humor del grupo etáreo III}




\section{(61 años)}

Hay un guajiro que va de viaje a La Habana y al lugar que él va llega a una tiende que venden pájaros/ y le llama la atención una cotorra que valía 200 dólares/ y le pregunta al hombre de la tienda qué tenía de especial esa cotorra// El hombre de la tienda le dice que esa cotorra pone los huevos cuadrao/ y el guajiro de nuevo le dice/ qué hago yo con una cotorra así si ni canta ni habla// Dice el dependiente/ que no/ cada ve que va a poner un huevo dice cCCOOOÑÑÑOOOO.......//

\section{(71 años)}

Va la vieja a visitar una de sus hijas y cuando llega se la encuentra completica en cuera/ y le dice ofendida/ pero por qué estás así/ qué manera esta de recibir a tu madre/ la muchacha le responde/ este es el traje del amor/ tienes que estar a la moda mamá/ esta es la última moda// Ahí siguen conversando un rato y la vieja sale pa la casa de la otra hija y tremenda sorpresa/ cuando llega estaba igual en cueras/ ensañándolo todo/ y le dice pero tú también/ no lo puedo creer// La hija le responde rápido/ no te asombres mamá/ este es el traje del amor/ así los maridos quieren más a uno/ esto es una maravilla// Cuando la vieja se fue pa la casa llevaba la idea del traje del amor/ pensando en darle una sorpresa a su viejo/ lo estaba esperado desnuita en pelota/ y el viejo asombrao le dice:

- Pero vieja/ qué te ha pasao/ qué tú haces así sin ropa//

- No/ viejo este es el traje del amor//

- Será el traje del amor/ pero plánchalo// 